\title{
Understanding the drivers for the development of design rulles for the synthesis of cylindrical flexures
}

\author{
M. J. Telleria and M. L. Culpepper \\ Massachusetts Institute of Technology, Cambridge, USA \\ Correspondence to: M. J. Telleria (mtelleri@ mit.edu)
}

Received: 28 February 2011 - Revised: 21 March 2012 - Accepted: 24 March 2012 - Published: 12 April 2012

\begin{abstract}
Cylindrical flexures (CFs), defined as flexures with only one finite radius of curvature loaded normal to the plane of curvature, present an interesting research direction in compliant mechanisms. CFs are constructed out of a cylindrical stock which leads to geometry, manufacturability, and compatibility advantages. Synthesis rules must be developed to design these new systems effectively. Current knowledge in flexure design pertains to straight-beam flexures or curved flexures loaded along the plane of curvature. CFs present a challenge because their mechanics differ from those of straight beams, and although their modelling has been researched thoroughly it has yet to be distilled into element and system creation rules. This paper uses models and finite element analysis to demonstrate that current design rules for straight-beam flexures are insufficient and inadequate for the design of CF systems. The presented discussion will show that CFs differ both at the element and systems levels, and therefore future research will focus on developing the three components of the building block approach: (i) reworking of element mechanics models to reveal the parameters which cause the kinematics of the curved beam to differ from those of the straight beam, (ii) development of a visual stiffness representation, and (iii) formation of system creation rules.
\end{abstract}

\section{Introduction}

Cylindrical Flexures, CFs, are defined as flexure systems with elements that have only one finite radius of curvature and are loaded normal to their plane of curvature. In other words, systems composed of flexure beams that are curved in a single plane. Figure 1 shows a prototype of a particular CF system. The system shown in Fig. 1 is actuated by loading the flexures normal to their plane of curvature. This specific loading condition is presented because it offers the most challenging research aspects and it is the least studied.

Past research has given different names to flexures that fall under this definition. The most applicable definition is Smith's "hinges of rotational symmetry", which he defines as flexures constructed from solids of revolution (Smith, 2000). The flexure shown in Fig. 1 fits within Smith's definition because of its axial symmetry. This definition is expanded to allow CFs to be fractions of a cylinder. The work is scoped by constraining CFs to elements with a single finite radius. Finally the system must have well defined distortions for it to be classified as a CF. The focus of this paper is to demonstrate: (i) the usefulness of design rules in the design process, (ii) that current design rules for straight-beam flexures are insufficient and inadequate for the design of systems with curved-beam flexures, (iii) the need for future research at the element and systems level, to develop guidelines for the design of CF systems.

\subsection{Prior art}

Past work that pertains to CFs has focused on two areas: (i) models for curved beams and (ii) analysis of specific $\mathrm{CF}$ concepts. Curved beams loaded normal to their plane of curvature have been studied for over $80 \mathrm{yr}$, leading to closedform expressions of their mechanics and dynamics (Timoshenko, 1930; Young and Budynas, 2002; Lee, 1969). These models, however, have not been distilled to provide a designer with guidelines as to how to use curved beams to achieve a set of functional requirements. This work will extract, from these analytical models, clear rules for both element and system design. An example of this kind of 


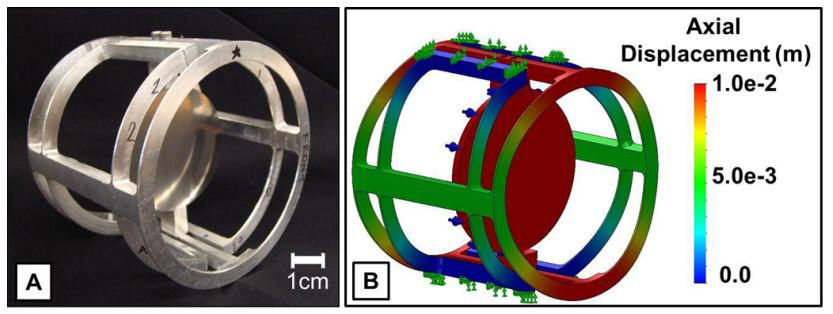

Figure 1. The quad compound-spring cylindrical flexure, CF, example: (A) 7075Al prototype and (B) FEA model depicting its actuation.

synthesis is the work by Kim et al. (2008), which uses curved beam building blocks (CBB) to create flexure systems. This paper differentiates itself from $\mathrm{CBB}$ in that in this case the beams are loaded normal as opposed to parallel to the plane of curvature. This loading condition requires the analysis of the flexures in three dimensions.

The other area of prior art pertains to the analysis of specific systems that fit within the CF definition. Smith (2000) presents detailed analysis on the disc coupling and the rotationally symmetric hinge. These types of analyses have produced useful flexure systems; however there has been little overarching insight developed that could be used to create new CF concepts. In many cases the analysis of these systems has relied on FEA given the lack of knowledge on what parameters determine the system performance. The biggest knowledge gap comes in the form of understanding how to assemble these curved elements to create predictable systems.

The lack of design guidelines restricts the design process. The rapid generation of concepts is limited, since the designer does not have a simple way to predict the general behavior of a system composed of CF elements. In addition optimization is tedious because there is little understanding of the effect of different parameters.

\subsection{Advantages of CFs}

Cylindrical flexures present geometry, manufacturability, and compatibility advantages over traditional flexure systems. Their axial symmetry may be used to achieve insensitivity to thermal changes, and to decrease the effects of manufacturing and load placement errors. Monolithic systems with a variety of flexure elements can be created out of a single piece of round stock reducing assembly cost and errors.

CF fabrication is facilitated by the availability of accurate round stock. CFs can be manufactured at low cost by using traditional machining methods. The prototype in Fig. 1 was machined using a 4-axis Mazak brand lathe. Other manufacturing methods include: a waterjet with a rotary axis and a 5-axis mill. CF's most attractive quality is their compatibility with rotating applications, laparoscopic tools, optical
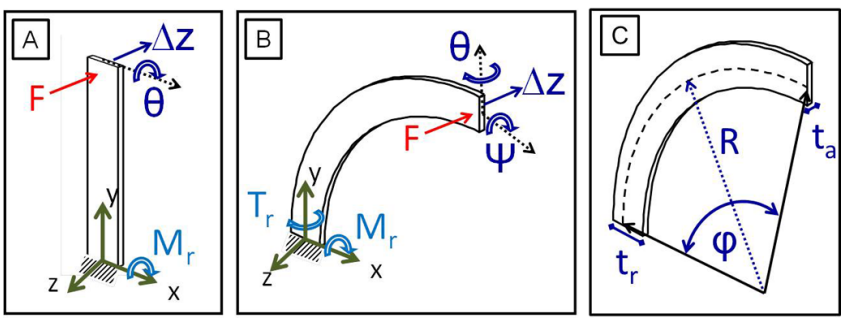

Figure 2. (A) Straight-beam mechanics, $F$ is the load on the beam, $M_{\mathrm{r}}$ is the resulting bending moment, $\Delta z$ is the displacement along the $\mathrm{z}$-axis, and $\theta$ is the parasitic rotation about the $\mathrm{x}$-axis. (B) Curved-beam mechanics, highlighting the added twist, $\psi$, and torque, $T_{\mathrm{r}}$ and $(\mathbf{C})$ Curved-beam parameters. $R$ represents the radius of curvature, $\phi$ is the sweep angle, $t_{\mathrm{r}}$ is the thickness of the beam in the radial direction, and $t_{\mathrm{a}}$ is the thickness in the axial direction.

systems, and other applications benefiting from their cylindrical geometry.

\section{Knowledge gap: need for CF research}

This section focuses on explaining how and why CFs behave differently than straight-beam flexures. These differences make current design guidelines inadequate for the effective creation of $\mathrm{CF}$ concepts. The variations from straightbeam behavior are illustrated by looking at a curved cantilever beam loaded at its free end. In this case the desired motion is a displacement along the $\mathrm{z}$-axis. All other displacements are defined as parasitic motions.

\subsection{Mechanics}

Curved flexures have additional complexities over straight beams both at the element and system levels. The curvature of the beam leads to an added rotation and resulting torque. Figure 2 shows that for a given load, $F$, the flexure will twist, $\psi$, which leads to a resulting torque, $T_{\mathrm{r}}$, at the base. Sect. 3.1 presents the proposed process for developing element design rules from current curved-beam models.

The curvature of the beam also leads to challenges in the conceptual assembly of curved-beam flexure systems. The traditional rules for adding elements have to be augmented to include the effects of the added twist and torque. The fourbar linkage and Jones et al.'s (1956) compound rectilinear spring are used to demonstrate the need for additional system creation rules.

A common flexure bearing system is the four-bar linkage, shown in Fig. 3a, where a stage is connected to ground through 2 parallel straight beams or blades. Figure 3a shows that the four-bar linkage's motion can be described as the super position of two deflections which lead to two parasitic motions in addition to the desired displacement along the $\mathrm{z}$ axis. Slocum (1992) gives the equations used to calculate the parasitic pitching motion of the stage, $\theta_{\text {pitch }}$, and estimate the 

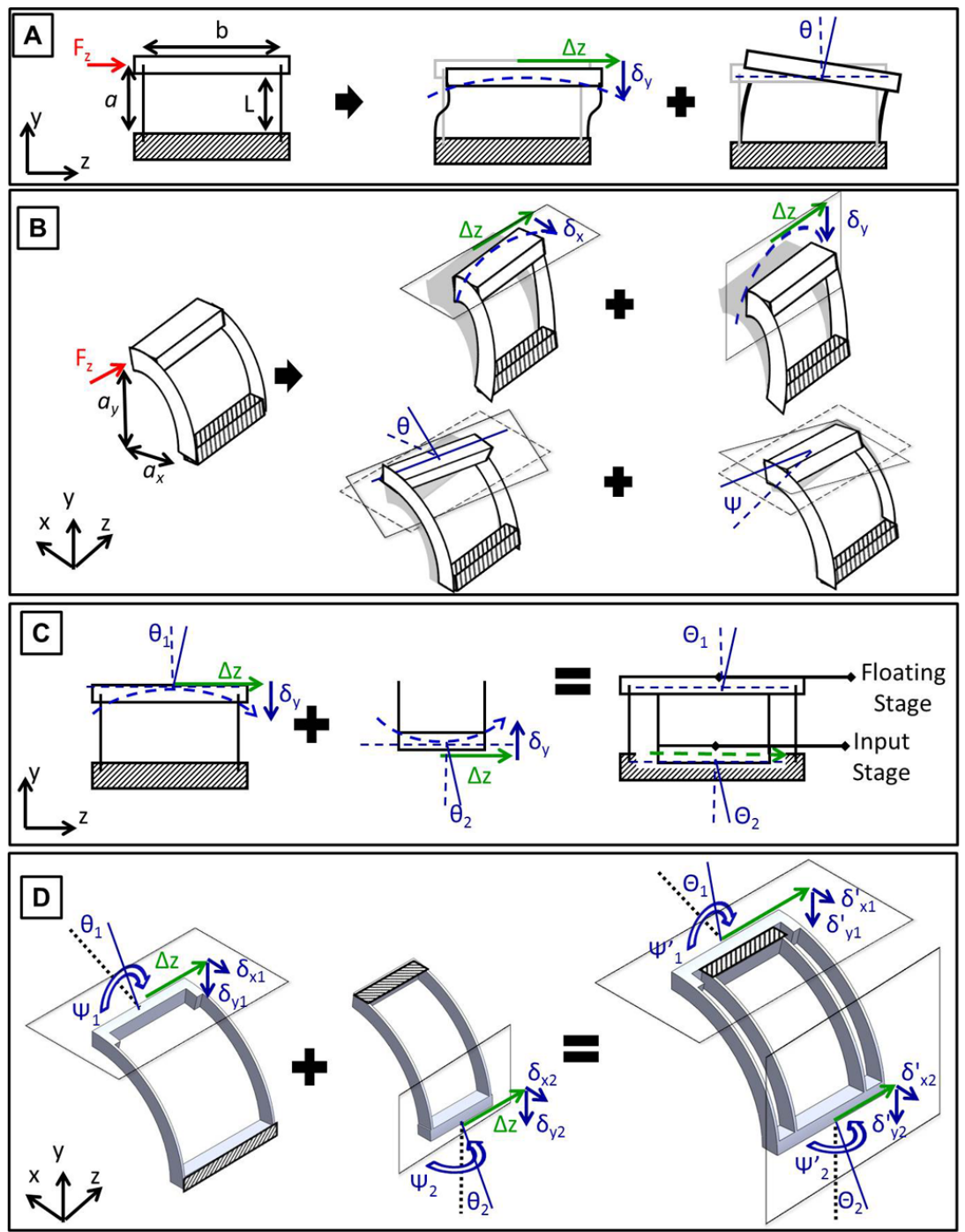

Figure 3. (A) Straight four-bar linkage parameters, $a$ indicates the location of $F_{z}$ relative to ground. Figure shows the three motions associated with $F_{z}$ applied onto the platform. Desired displacement $\Delta z$ is indicated with green, while the parasitic motions, $\theta$ and $\delta_{y}$, are shown in blue. (B) Curved four-bar linkage parameters and displacements under $F_{z} ; a_{y}$ and $a_{x}$ indicate the location of the force relative to ground. The curvature results in two $\delta$ parasitic motions, $\delta_{x}$ and $\delta_{y}$, and an additional rotation of the stage, $\Psi$. (C) Straight compound-spring. Nesting of the two four-bars results in cancellation of the $\delta$ parasitic motions. $\Theta_{1}$ and $\Theta_{2}$ indicate the rotations of the stages in the nested system. (D) Curved compound-spring. Cancellation of the $\delta$ motions is not straight forward.

vertical motion, $\delta_{y}$, presented in Eqs. (1) and (2). In these equations $L$ represents the length of the flexure beam, $b$ is the distance separating the flexures, $a$ is the distance from the load input location to ground, $t$ is the thickness of the beams, and $\Delta z$ is the desired displacement along the load direction. The parameters are defined in Fig. 3a. The vertical motion of the stage, $\delta_{y}$, in conjunction with the desired displacement, $\Delta z$, will result in the stage following an $\operatorname{arc}$ as $F_{z}$ is applied, as highlighted in Fig. 3a.

$$
\begin{aligned}
& \theta_{\text {pitch }}=\left(\frac{6(L-2 a) \cdot t^{2}}{3 b^{2} L-2 t^{2}+6 a t^{2}}\right)\left(\frac{\Delta z}{L}\right), \\
& \delta_{y} \approx \frac{(\Delta z)^{2}}{2 L} .
\end{aligned}
$$

Equations (1) and (2) are used by designers to estimate the parasitic motions of a straight four-bar system. These equations have been derived for straight-beam flexures and must be expanded in order to describe a four-bar composed of curved beams. Figure $3 \mathrm{~b}$ shows that for an input load normal to the plane of curvature, $F_{z}$, the motion of the input stage can be decomposed into four deflections which lead to four parasitic motions: $\theta, \Psi, \delta_{y}$ and $\delta_{x}$. As a result of the 
curvature of the flexures, the $\mathrm{CF}$ four-bar the stage will travel in two arcs dictated by $\Delta z, \delta_{y}$ and $\delta_{x}$, and it will experience two rotations about its center $\theta, \Psi$. Future research is necessary in order to develop a set of equations that accurately predict the parasitic motions of the four-bar CF system.

The design of precision machines requires that the parasitic motions are well understood and minimized to achieve the desired displacement. Studies have shown that for a fourbar linkage load placement and nesting of systems can be used to reduce the parasitic motions. As Eq. (1) shows $\theta_{\text {pitch }}$ can be reduced by moving the location of the input load, $a$. The challenge in the curved version of the four-bar linkage is that the load location is now defined by two coordinates $a_{x}$ and $a_{y}$, as shown in Fig. 3b. Once again the currently available knowledge is insufficient to design a curved-beam flexure system.

Nesting of two four-bar flexure systems, referred to as the compound rectilinear spring, has been used to mitigate the parasitic arcing motion caused by $\delta_{y}$ as shown in Fig. 3c. In the compound-spring the $\delta_{y}$ motion of the input stage is matched by an equal and opposite $\delta_{y}$ of the floating stage resulting in a cancellation of the arcing motion of the input stage. The $\delta_{y}$ motions are mitigated as long as both four-bars are constructed of identical flexure beams, therefore achieving the same $\Delta z$ displacement and as a result the same magnitude of $\delta_{y}$. It is important to note that the nesting of the flexures does not remove the parasitic rotations of the stages, $\theta_{1}$ and $\theta_{2}$, but these rotations are affected by the nesting and are labelled $\Theta_{1}$ and $\Theta_{2}$ in Fig. 3c to reflect this. Figure 3d shows what happens when a compound-spring is created using two curved four-bars. Due to the curvature, the floating and input stages are located on different planes. As a result the cancellation of the $\delta$ motions is not straight forward and requires further research; the resulting $\delta$ motions will depend on the magnitude of $\delta_{y}$ and $\delta_{x}$ as well as the sweep angle, $\phi$. Section 3.3 discusses the proposed research approach for creating system rules that will allow the designer to manage the parasitic motions of CF systems.

\subsection{Stress}

In order to determine the range of a system, both the displacement to a given load and the resulting stress must be considered. The curvature of the element leads to a torque on the fixed end of the cantilever beam. This torque will affect the resulting stress calculation. Using energy conservation principles it can be identified that an added torque requires that there be a decrease in the original resulting moment. Both of these effects will change the magnitude and distribution of the stress along the beam.

Figure 4 shows that a stress concentration is observed at the base on the inner radius of the curved element. Future work will develop guidelines that will allow the designer to account for the new stress distribution and perhaps find a way to distribute the stress more evenly. Previous work has shown

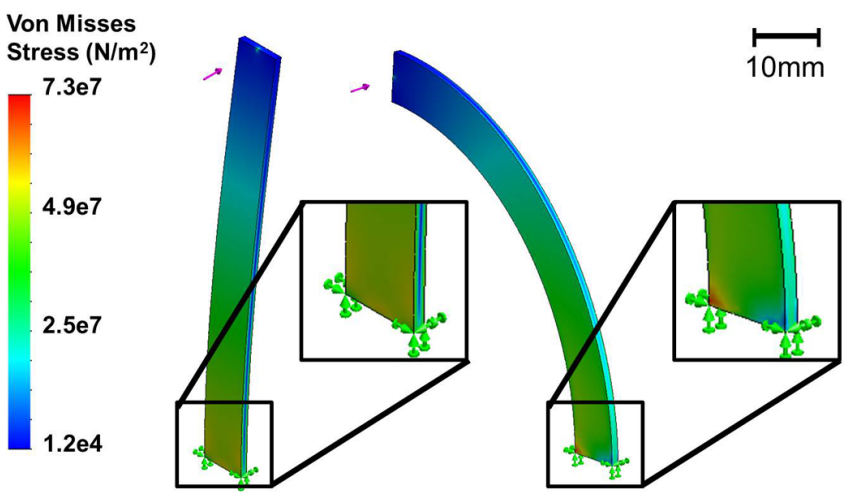

Figure 4. Finite element analysis of two cantilevered beams of the same properties under the same loading force. The stress concentration on the curved beam is highlighted.

contouring of the thickness of the flexure to be a successful way to distribute stress along the length of a beam (Timoshenko, 1930).

\section{Research approach and impact}

The main three compliant mechanism synthesis approaches are topology synthesis, pseudo-rigid modelling, and building block approach. A building block approach derived from constraint-based design is proposed as the most appropriate synthesis methodology for CFs at this time (Maxwell, 1890; Blanding, 1999; Hale, 1999). The plan is to first develop a full understanding of the parameters that affect the element's behavior. This element then becomes the building block for CF systems. The next step is to develop rules for how these blocks interact when added together. The desired outcome of the research consists of (i) a visual representation that allows the designer to quickly understand how different parameters will affect the behavior of an element, similar to a stiffness ellipsoid (Kim, 2008) and (ii) design guidelines for the generation of CF systems.

A constraint-based design approach has been chosen because it is intuitive for precision engineers. This synthesis approach presents a quick way to understand why a system created from a set of building blocks will behave a certain way. It also makes the rapid concept generation phase of the design process very efficient as the designer can use the stiffness representations and system rules to quickly lay out the elements to achieve a desired performance, while being able to account for external constraints such as manufacturing. The knowledge gathered through the development of these building block rules can then be used to create arguments for the other synthesis methods. 
A
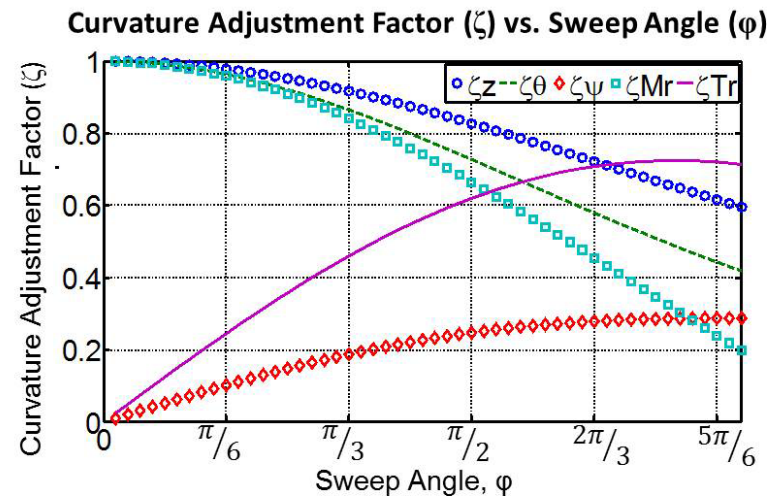

B

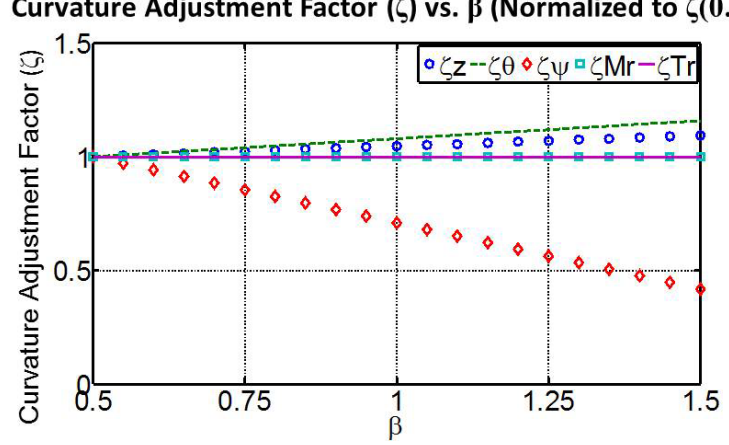

Figure 5. The curvature adjustment factors, $\zeta$, show how the behavior of the curve beam deviates from that of a straight beam as a function of the geometry of the beam. Curvature adjustment factors are obtained from Roark's closed-form solutions. $\zeta_{z}$ corresponds to the z-axis displacement multiplier as shown in Eq. (7), while $\zeta_{\theta}$ and $\zeta_{\Psi}$ correspond to the multipliers for the two parasitic motions $\theta$ and $\Psi$, respectively. Finally $\zeta_{M_{\mathrm{r}}}$ and $\zeta_{T_{\mathrm{r}}}$ represent the curvature adjustment factors for the resulting moment and torque at the base of the beam. Flexure length is held constant by varying the radius of curvature. (A) Curvature adjustment factors, $\zeta$, vs. sweep angle, $\phi$. The graph shows that as the sweep angle goes to zero the curved beam behaves as a straight beam, $\zeta_{z}, \zeta_{\theta}, \zeta_{M_{\mathrm{r}}}$ go to 1 , and $\zeta_{\Psi}$ and $\zeta_{T_{\mathrm{r}}}$ approach zero. While as the sweep angle increases the behavior of the curved beam deviates from that of the straight beam. (B) Curvature adjustment factors, $\zeta$, vs. $\beta, \beta$ is the ratio of elastic to shear properties of a curved beam as defined by Eq. (4).

\subsection{Element mechanics}

The first step in the building block approach is to understand the flexure element mechanics. The goal is to identify what parameters affect the element's performance. Roark's equations for curved beams loaded normal to their plane of curvature are used to understand the kinematics of the curved beam and to find the parameters that play an additional role in the motion of the curved beam (Young and Budynas, 2002). Cantilever beams loaded at their free end are first examined. Future work will explore the effect of load location.

Figure $2 \mathrm{c}$ shows the different parameters that define the curved flexure element. Roark presents equations for the displacements resulting from a load, $F$, at the free end of the beam (Young and Budynas, 2002). The z-displacement, $\Delta z$, is the desired motion. This displacement is given by Eq. (3), where $R$ is the radius of the beam, $E$ is the elastic modulus, $I$, is the second moment of area, and $\phi$ is the sweep angle. $C_{a 6}$, $C_{a 9}, C_{a 3}$ are functions of $\phi$ and $\beta$, defined in Roark (Young and Budynas, 2002). Equation (4) defines $\beta$ as the ratio of elastic to shear properties of the beam, where $G$ is the shear modulus, and $k$ is a torsional stiffness constant. More details on these equations can be found in Roark (Young and Budynas, 2002). Equations (5) and (6) from Roark are used to calculate the two parasitic motion of the cantilever beam, $\theta$ and $\Psi$ which are defined in Fig. $2 b$.

$$
\begin{aligned}
& \Delta z=\frac{F R^{3}}{E I} \cdot\left(C_{a 6} \sin (\varphi)-C_{a 9}(1-\cos (\varphi))-C_{a 3}\right), \\
& \beta=\frac{E \cdot I}{G \cdot k} . \\
& \theta=\frac{F \cdot R^{2}}{E \cdot I}\left(C_{a 6} \cos (\varphi)-C_{a 9} \sin (\varphi)\right) \\
& \Psi=\frac{F \cdot R^{2}}{E \cdot I}\left(C_{a 9} \cos (\varphi)+C_{a 6} \sin (\varphi)\right)
\end{aligned}
$$

It is proposed that a more efficient way to look at Eq. (3) is to factor it into the straight-beam equation and a curvature adjustment factor, $\zeta_{z}$, as shown in Eq. (7). In Eq. (7) the length, $L$, is the product of $R$, and $\phi$. Equation (8) shows that the curvature adjustment factor is calculated by dividing the displacement given by Roark in Eq. (3) by the straight-beam displacement equation. The curvature adjustment factors for the parasitic motions, $\zeta_{\theta}$ and $\zeta_{\Psi}$, are similarly calculated.

$$
\begin{aligned}
& \Delta z=\zeta_{z} \frac{F L^{3}}{3 E I}, \quad L=R \cdot \varphi \\
& \zeta_{z}=3 \cdot\left(C_{a 6} \sin (\varphi)-C_{a 9}(1-\cos (\varphi))-C_{a 3}\right) / \varphi^{3}
\end{aligned}
$$

This new representation allows the designer to use all previous knowledge of straight-beam behavior and then evaluate what parameters play an additional role in the behavior of the curved beam. The added role of parameters is evaluated by identifying their effect on $\zeta_{z}$. If $\zeta_{z}$ does not depend on the parameter then that parameter does not have an additional effect, relative to its effect on a straight beam's mechanics. If $\zeta_{z}$ is a function of that parameter then the parameter plays an additional role. Analysing the parameters in Eq. (1) it is found that $\zeta_{z}, \zeta_{\theta}$, and $\zeta_{\Psi}$ depend only on $\phi$ and $\beta$.

The next step is to understand how these two parameters affect the mechanics of the beam. Figure 5a shows how as $\phi$ approaches zero the curved beam behaves like a straight beam. Then as $\phi$ increases the behavior deviates. Figure $5 b$ shows the curvature adjustment factor for each of the motions $\zeta_{z}, \zeta_{\theta}$ and $\zeta_{\Psi}$, vs. $\beta$. The value of $\beta$ for a given geometry varies only a small amount with different materials because the elastic and shear modulus are related by the Poisson ratio, which is close to 0.3 for common flexure materials. Having identified the two additional parameters necessary to describe the kinematic behavior of the curved beam, future 


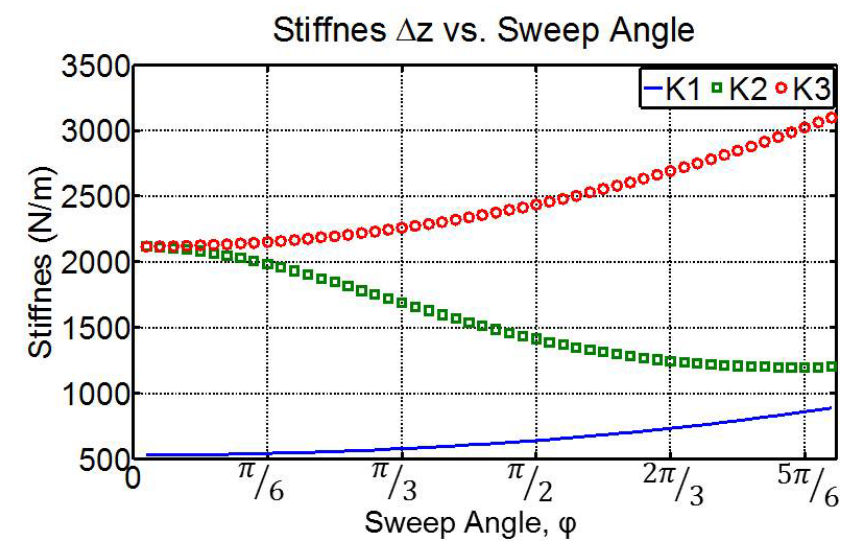

Figure 6. $\Delta z$-stiffness vs. sweep angle, $\phi$, for three constraint conditions. $K 1$ corresponds to a cantilever beam, $K 2$ represents a fixed end-slope constrained beam, and $K 3$ corresponds to a fixed endslope and twist constrained beam. Flexure length is held constant by varying the radius of curvature.

research will analyse how the sweep angle, $\phi$, and $\beta$ affect common flexure performance metrics.

\subsection{Stiffness representation}

Once the mechanics are understood, a stiffness representation can be developed. This representation will allow the designer to quickly identify how the elements will behave. This can be done using either stiffness or compliance ellipsoids (Kim et al., 2008). A stiffness ellipsoid visually shows the relative magnitude of the stiffnesses of a beam. The $\Delta z$ stiffness of the beam depends on the loading conditions and element constraints. The previous analysis focused on cantilevered beams; however, flexures are usually constrained in systems. Therefore, the effect of different constraint conditions on the $\Delta z$-stiffness is explored. The change in $\Delta z$ stiffness with $\phi$ is evaluated, because sweep angle is the dominant parameter.

Figure 6 shows the $\Delta z$-stiffness vs. $\phi$ for three different constraint conditions: (i) cantilever beam, $K 1$, (ii) slope constrained, $K 2$, and (iii) slope and twist constrained, $K 3$. The length of the flexures is held constant in this analysis by varying the radius of curvature. The graph for K1 corresponds to the cantilever beam analysed in Fig. 5. From the mechanics analysis $K 1$ is expected to increase with $\phi$, given $\Delta z$ decreases with $\phi . K 2$ decreases with $\phi$ because both $\Delta z$ and $\theta$ decrease with $\phi$. This decrease in slope translates to a smaller moment being applied at the free end to achieve the zero slope condition. Finally $K 3$ increases with $\phi$. This same analysis will be carried out for all stiffnesses leading to the creation of the stiffness ellipsoids.

The presented equations of motion for the curved cantilever beam analysed in Figs. 5 and 6 are corroborated using FEA models. Figure 7 plots the predicted displacements

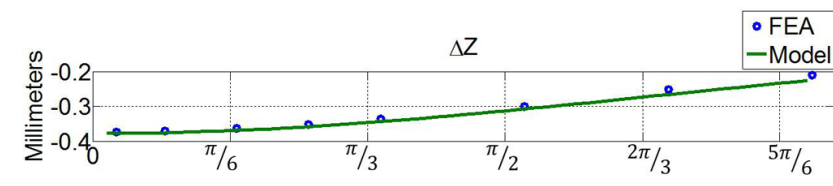

$\theta$

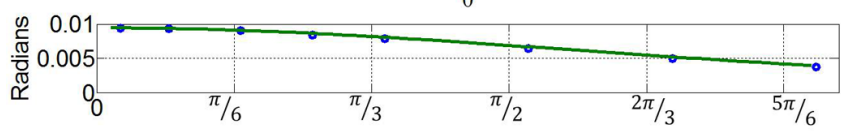

$\psi$

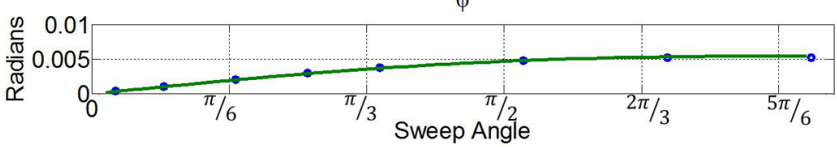

Figure 7. Comparison of the FEA and closed-form equation calculations of the curved beam displacements vs. sweep angle, $\phi$, for a cantilever beam, $K 1$. Max error between models is $7 \%$. $\Delta z$ represents the desired motion along the $\mathrm{z}$-axis of the beam as indicated in Fig. 2b. $\theta$ and $\Psi$ correspond to the two parasitic rotations of the beam under a load normal to the plane of curvature. Flexure length is held constant by varying the radius of curvature.
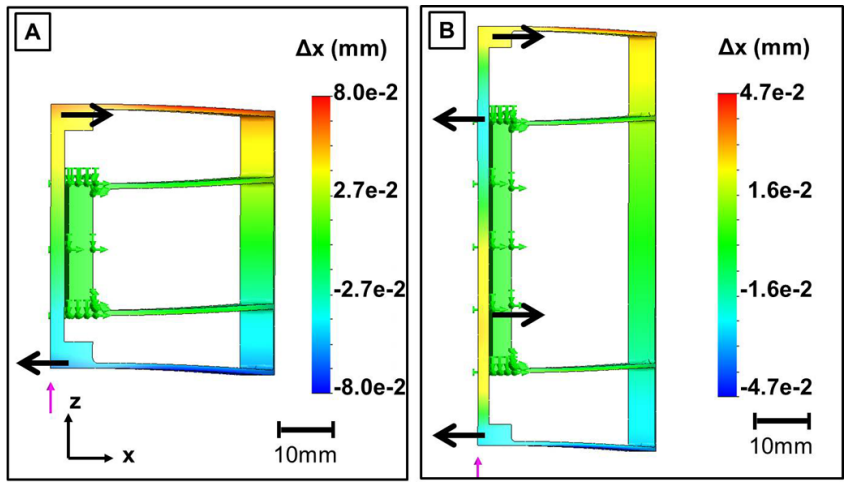

Figure 8. FEA of a curved compound-spring under a $10 \mathrm{~N}$ load (A) No deformation of input stage (B) Deformed input stage as a result of the four-bar twist stiffness, $K_{\psi}$, being larger than the stage stiffness.

from both models. The next step will be to compare these models to experimental results. Future research will expand on the kinematic analysis by assembling the $6 \times 6$ stiffness matrix for a curved beam.

\subsection{System analysis}

The system creation guidelines will focus on the interaction between the element building blocks. With these rules the designer will be able to assemble the blocks together to achieve a desired system performance. Three main system creation research areas have been identified so far: (i) the effect of element separation, (ii) the importance of the sweep angle, which leads to the ground and stage of the flexure system to be located on two different planes, and (iii) the effect of load location. 

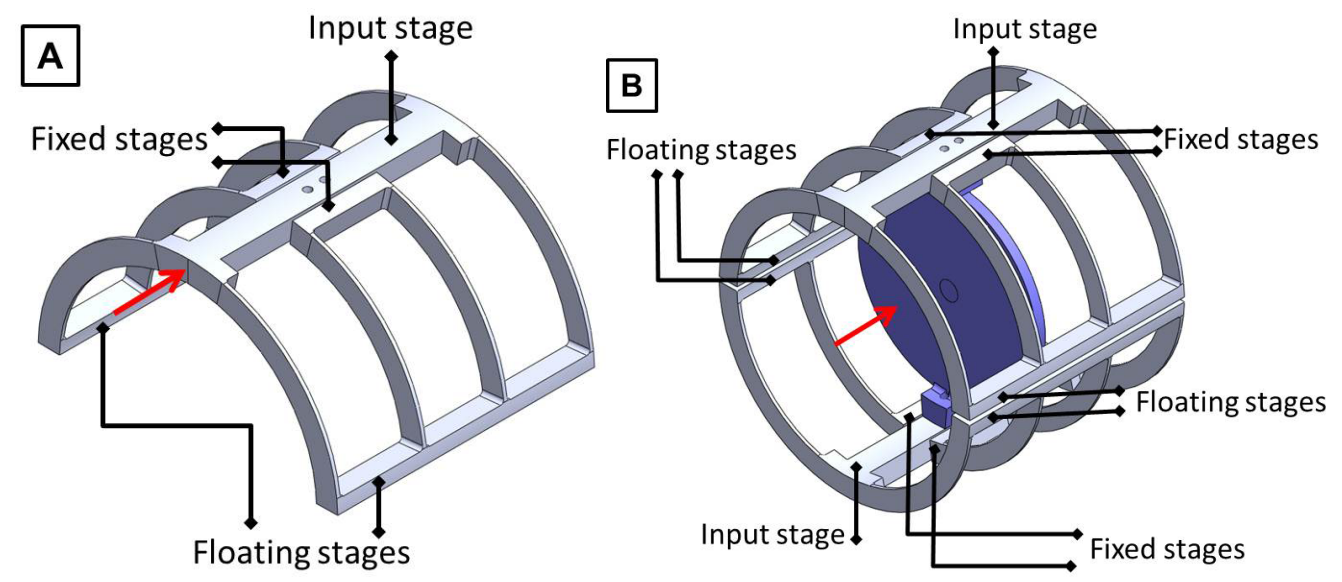

Figure 9. (A) Double curved compound-spring. (B) Stage driven by two separate double curved compound-springs, the quad compoundspring CF design.

Table 1. Main stage tip and tilt angle in microradians per millimeter of z-axis displacement for the different compound-spring iterations (flexure length is constant for all iterations).

\begin{tabular}{|c|c|c|}
\hline & 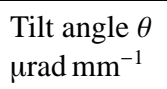 & 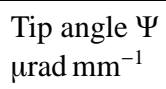 \\
\hline Flat straight compound & 58.5 & 0.00 \\
\hline $90^{\circ}$ curved compound & 321 & 101 \\
\hline Double curved compound & 0.00 & 211 \\
\hline Two double curved compound & 0.00 & 5.04 \\
\hline
\end{tabular}

Element spacing plays a critical role in system creation. It is well-established that parasitic rotations of a shaft decrease with the distance between the bearings, $b$, squared. Equation (1) shows that the pitching motion of a four-bar decreases with $b^{2}$ and Eq. (9) presents that the twist stiffness, $K_{\psi}$, of a straight four-bar increases with $b^{2}$ (Smith, 2000).

$K_{\psi}=\frac{E I}{b^{2} L}$,

For straight-beam systems both parasitic motions decrease with $b^{2}$. Using only this information may lead the designer to maximize the spacing between the flexure beams. This is not only a problem in terms of weight and volume. It is also found that since the parasitic motions have not been mitigated, when the four-bar's spacing is large enough, $K_{\psi}$ is greater than the stiffness of the stages and the stages begin to deform, as shown in Fig. 8. Increasing the spacing between the beams is not a complete solution for reducing the parasitic motions.

Load location has been shown to play a critical role in reducing the parasitic motions of a system (Slocum, 1992; Hopkins, 2010). Therefore, future research must establish rules and equations that describe the effect of the load location on the parasitic motions of a curved system. The re- search approach proposed is to first establish the optimal load location for a curved-beam element and how this position changes with sweep angle. Then to determine how to use center of stiffness rules to find the best actuation point of a CF system.

Section 2.1 showed that a curved four-bar system has four parasitic motions and postulated that in a curved compoundspring the $\delta$ motions of the stage are not mitigated through the nesting because they occur on two different planes and have different $\delta_{x}$ and $\delta_{y}$ magnitudes. The question then becomes how do to deal with the additional parasitic motions and the fact that they are occurring on two different planes. The following example is used as a way to demonstrate some of the challenges of CF system creation. The goal of the presented system is to translate a stage along the CF's centralaxis with minimal tip and tilt error. To do this the parasitic $\theta$ and $\Psi$ motions of the flexure system have to be minimized and the $\Delta z$ displacement maximized. Precision engineering applications require high accuracy flexure systems; microns of parasitic motion can cause a design to fail.

To improve the performance of the curved version, a double compound-spring is used, as shown in Fig. 9. This design removes the tilt angle, $\theta$, of the input stage by using symmetry without severely over-constraining, given the side/floating stages are free to pitch and tilt. Symmetry can be used once again to reduce the tip angle, $\Psi$, by driving a stage with two double curved compound-springs as shown in Fig. 9. The displacements of the different iterations are compared to the compound rectilinear spring in Table 1. Finally the side stages have to be connected to achieve a full cylinder in order to have a monolithic system. The challenge is to ensure that the side stages are able to continue to move relative to each other. The stages can be connected through a flexure spring that allows for this relative motion. 


\section{Conclusions and future work}

This paper uses analysis of common flexure mechanisms to demonstrate that current design rules for straight-beam flexures are insufficient for designing effective CFs. The insights that this research has already generated guided the correction of a CF design achieving a $20 \times$ reduction in parasitic errors. The result is a new CF concept that is compatible with cylindrical geometries. Continuing research in this area will develop a full set of design insights and a stiffness representation that will enable a designer to create CF systems effectively.

Acknowledgements. This work was supported in part by the National Science Foundation Graduate Fellowship Program and Lincoln Laboratories. Special thanks to MIT's BioInstrumentation lab for the use of their Mazak Lathe.

Edited by: J. Herder

Reviewed by: B. Trease and D. Brouwer

\section{References}

Blanding, D. L.: Exact Constraint: Machine Design Using Kinematic Principles, ASME Press, New York, 1999.

Hale, L. C.: Principles and Techniques for Designing Precision Machines, Ph.D. thesis, Massachusetts Institute of Technology, USA, 1999.
Hopkins, J. B.: Design of Flexure-Based Motion Stages for Mechatronic Systems via Freedom, Actuation and Constraint Topologies (FACT), Ph.D. thesis, Massachusetts Institute of Technology, 2010.

Jones, R. V., Phil, D., and Young, I. R.: Some parasitic deflexions in parallel spring movements, Scientific Instruments, 33, 11-15, 1956.

Kim, C. J., Moon, Y., and Kota, S.: A building block approach to the conceptual synthesis of compliant mechanisms utilizing compliance and stiffness ellipsoids, Mech. Design, 022308-1002308-11, 130, 2008.

Lee, H.: Generalized Stiffness Matrix of a Curved-Beam Element, AIAA Journal Technical Notes, 7, 2043-2045, 1969.

Maxwell, J. C.: General considerations concerning scientific apparatus, The scientific papers of James Clerk Maxwell, edited by: Niven, W. D., Dover Press, (Reprinted from the handbook to the special loan collection of scientific apparatus, 1876), 1890.

Slocum, A. H.: Precision machine design, Society of Manufacturing Engineers, Society of Manufacturing Engineers, PrenticeHall, Inc., USA, 1992.

Smith, S. T.: Flexures: Elements of elastic mechanisms, CRC Press, USA, 2000.

Timoshenko, S.: Strength of materials, D. Van Nostrand, USA, 1930.

Young, W. C. and Budynas, R. G.: Roark's Formulas for Stress and Strain, McGraw-Hill, Singapore, 2002. 